\title{
Stability for a Class of State Constrained Impulsive Nonlinear Systems: Barrier Lyapunov Functions Method
}

\section{Liangliang Li ( $\sim$ liliangliangll|@126.com )}

Huaibei Normal University https://orcid.org/0000-0002-2534-4286

\section{Zhengwen Tu}

Chongqing Three Gorges University

Guanghui Zhou

Huaibei Normal University

\section{Research Article}

Keywords: State constrained, Impulsive systems, Barrier Lyapunov functions, Nonlinear systems, Stability

Posted Date: August 13th, 2021

DOI: https://doi.org/10.21203/rs.3.rs-796093/v1

License: (c) (i) This work is licensed under a Creative Commons Attribution 4.0 International License.

Read Full License 


\title{
Stability for a class of state constrained impulsive nonlinear systems: Barrier Lyapunov Functions Method
}

\author{
Liangliang $\mathbf{L i}^{1} \cdot$ Zhengwen $\mathrm{Tu}^{2} \cdot$ Guanghui $\mathrm{Zhou}^{1}$
}

Received: date / Accepted: date

\begin{abstract}
This paper studies the problem for a class of state constrained impulsive nonlinear systems. Firstly, we establish two sufficient conditions for the stability of invariant sets of state constrained hybrid systems. Secondly, we construct the symmetric and asymmetric barrier Lyapunov functions, respectively. A feedback method is presented to solve the stabilization problem of constrained hybrid systems. Introduce the auxiliary matrix, combining with inductive method and linear matrix inequality theory, some sufficient conditions are obtained to ensure stability for state constrained hybrid dynamical networks by the attractive ellipsoid method approach. Finally, one example with simulations is given to validate the effectiveness of the proposed criteria.
\end{abstract}

Keywords State constrained - Impulsive systems · Barrier Lyapunov functions · Nonlinear systems · Stability

\section{Introduction}

Input constraints and output constraints are existing extensively in nonlinear systems $[1,2]$. Input constraints are caused by the structure, material and motion characteristics of the physical system, including delays, dead zone [3] and saturation etc. And the states of the nonlinear system may be restricted. For example, in robot control, there is a maximum torque in the motor control, that is actuator saturation. It maybe led the robot

\section{L.L. $\operatorname{Li}(\otimes)$}

e-mail: lilianglianglll@126.com

1 School of Mathematical Sciences, Huaibei Normal University, Huaibei, Anhui 235000, China.

2 School of Mathematics and Statistics, Chongqing Three Gorges University, Chongqing, 404100, China loses part of the control signal, which may make the robot deviate from the predetermined trajectory, or even cause a safety accident [4]. In the control system of a car, the movement of the wheel has a maximum speed, that is, the output signal of the system is limited within a specific range. If the influence of these nonlinear characteristic is ignored in the design of the control system, the whole nonlinear system may be unstable, and even damage the whole system. Therefore, it is necessary to solve the nonlinear system control problem with constraints in the field of nonlinear system control.

In many practical engineering and natural system$\mathrm{s}$, the state of the system changes abruptly at certain points in time for some reason. That is the mutation or jump process can be regarded as happening at a moment. These systems can't be solved by traditional continuous systems or discrete systems alone. Such as population growth in ecology [5], prevention and treatment of infectious diseases, digital communication systems, optimal control in economics(economic stimulus package) [6], and so on. This phenomenon can also be found in many engineering fields, such as automatic control, computer networks, supply chain systems, and communication systems [7]. A system in which the state suddenly changes at some moment cannot be described as a single continuous dynamical system or a discretetime dynamical system, so it is natural that people put forward an impulsive system to describe the dynamic system with impulsive phenomenon. In recent decades, many researchers have focused on stability or control of impulsive systems or hybrid systems, include Lyapunov stability of impulsive systems [8-12], impulsive control for synchronization of complex networks [13], application of hybrid systems $[14,15]$. Impulsive systems have been studied for a long time, but still less referred to the state constrained impulsive nonlinear systems. 
In conclusion, some researchers have attempted to solve the problem of constrained systems [16-29]. In [16], Rao et al. investigated moving horizon estimation as an online optimization strategy for constrained state estimation for nonlinear discrete-time systems. Harris et al. analyzed the problem of finite horizon optimal control with mixed non-convex and linear state constraints [17]. Recently, barrier Lyapunov function is proposed to solve the input or output constraint system [18, 19]. In [19], Dehaan and Guay considered a class of nonlinear systems with unknown dynamical parameters, whose states are subject to the unknown state-constrained minimizer with unknown parameters. Liu and Tong [24] studied a class of uncertain nonlinear parametric systems by an adaptive control technique and barrier Lyapunov functions. Tee et al. [25] considered the output tracking control for strict feedback delayed nonlinear systems with output constraint. In [26], a reinforcement learning control method is proposed for air-breathing hypersonic vehicles based on barrier Lyapunov functions. In [28], the authors considered the issue of the uncertain switched multi-input and output-constrained nonlinear systems by adaptive neural tracking control. However, although the aforementioned results account for the state constraints, the approaches cannot cope with the problem of the state constrained impulsive nonlinear systems. This key point motivates us to solve the problem. Based on this, this paper proposes a special type of feedback control with disturbance, which addresses the state constrained system with impulsive effects. The matrix theory and convex analysis method show that the state track for the closed loop nonlinear dynamic systems are stable. Finally, one instance with numerical simulations is offered to demonstrate the availability of our results.

This paper is organized as follows: In the following section, model and some theorem are presented. The robust control applications are established in Section 3. Simulation example is reported in Section 4, and the conclusion is drawn in Section 5.

Notations: Throughout this paper, $\mathbb{R}^{n}$ and $\mathbb{R}^{n \times m}$ denote the $n$-dimensional Euclidean space and the set of $n \times m$ matrices, respectively. $\mathbb{R}^{+}=(0,+\infty) . \mathbb{N}=$ $\{1,2, \cdots\} . A / B=\{x \mid x \in A, x \notin B\} . \partial S$ denotes the boundary of set $S$. Denote $\|u\|$ as a vector norm of the vector $u \in \mathbb{R}^{n} . C(\mathbb{X}, \mathbb{Y})$ denotes the space of continuous mappings from the topological space $\mathbb{X}$ to the topological space $\mathbb{Y}$. The notation $A^{\mathrm{T}}$ and $A^{-1}$ mean that the transpose of $A$ and the inverse of a square matrix $A$, respectively. $X>0(X \geq 0)$ means that $X$ is a real symmetric and positive definite(positive semi-definite) matrix. $I$ denotes the identity matrix.

\section{Model description and some preliminaries}

We consider the nonlinear dynamic systems described by the following hybrid differential equations:

$$
\left\{\begin{array}{l}
\dot{x}(t)=f(x(t)), \text { a.e. } t \geq 0, x(t) \in \mathbb{D}, \\
\triangle x(t)=S(x(t)), x(t) \in M \subset \partial\left(\mathbb{R}^{n} / \mathbb{D}\right), \\
x(0)=x_{0} \in \mathbb{D},
\end{array}\right.
$$

where $x(t) \in \mathbb{R}^{n}$ corresponds to the state at time $t, \mathbb{D}$ is a bounded domain in $\mathbb{R}^{n} \cdot f(x(t)) \in C\left(\right.$ mathbbD, $\left.\mathbb{R}^{n}\right)$. $M$ presents the impulsive point set. Assume that if there exists a $x\left(t_{k}\right) \in M$, note that $\triangle x\left(t_{k}\right)=x\left(t_{k}^{+}\right)-$ $x\left(t_{k}^{-}\right), x\left(t_{k}^{+}\right)=x\left(t_{k}\right)$ and $x\left(t_{k}^{-}\right)=\lim _{t \rightarrow t_{k}^{-}} x(t)$. Where $S(x(t))$ is a continuous single valued mapping. For system (1), its initial conditions are given by $x(0)=x_{0} \in$ $\mathbb{D}$.

The set $T_{0}$ is called an invariant set of system [30,31] if it follows from the inclusion $x_{0} \in T_{0}$ that $x\left(t, x_{0}\right) \in T_{0}$ for $t \geq 0$. We establish some sufficient conditions for the stability of invariant sets of state constrained hybrid systems.

Theorem 1. Let $\mathbb{D}$ is a bounded domain in $\mathbb{R}^{n}$, for any positive constants $\alpha, \mu_{k} \leq 1$, there exists a positive function $V(x)$ satisfies the following conditions:

$$
\left\{\begin{array}{l}
\left.D^{+} V(x)\right|_{(1)} \leq-\alpha V(x), x \in \mathbb{D} \\
V(x+S(x)) \leq \mu_{k} V(x), x \in M \subset \partial .
\end{array}\right.
$$

Then $x$ of system (1) remains in the open set $\mathbb{D}$.

Proof. In the set of all trajectories of system, we consider the following classes:

1): The collection of trajectories that do not have common points with the set $M$. That is, for a certain $x_{0}$ and $t \geq 0$, we have $x\left(t_{0}, x_{0}, t\right) \notin M$, the $t=+\infty$. From the fact that $x_{0} \in \mathbb{D}$ and $V\left(x_{0}\right)$. Since $V(x)$ is positive definite and $D^{+} V(x) \leq-\alpha V(x) \leq 0$, we obtain that $V(x) \leq V\left(x_{0}\right)$ for $t=+\infty$. This implies that the conclusion is true.

$2)$ : The collection of trajectories that have common points with the set $M$. Assume that the sequences $\left\{t_{k}^{x_{0}}\right\}, k=1,2, \ldots$. Assumed that $t_{k+1}^{x_{0}}>t_{k}^{x_{0}}$ and $t_{k}^{x_{0}} \rightarrow$ $+\infty$ as $k \rightarrow+\infty$.

For $t \in\left[0, t_{1}^{x_{0}}\right)$, and $D^{+} V(x) \leq-\alpha V(x)$. Then $V(t) \leq V\left(t_{0}\right) e^{-\alpha t}$ for $t \in\left[0, t_{1}^{x_{0}}\right)$.

Combining with $\mu_{1} \leq 1$ and (2), when $t=t_{1}^{x_{0}}$, then $V\left(t_{1}^{x_{0}+}\right) \leq \mu_{1} V\left(t_{1}^{x_{0}}\right) \leq \mu_{1} V\left(t_{0}^{-}\right) e^{-\alpha t_{1}^{x_{0}-}} \leq V\left(t_{0}\right)$.

By deduction method, then for $t \in\left[t_{k}^{x_{0}}, t_{k+1}^{x_{0}}\right)$, from $(2)$, and $V\left(t_{k}^{x_{0}+}\right) \leq \mu_{k} V\left(t_{k}^{x_{0}-}\right) \leq \mu_{k} V\left(t_{k-1}^{-}\right) e^{-\alpha t_{k}^{x_{0}-}} \leq$ $V\left(x_{0}\right)$.

Then $V(t) \leq V\left(x_{0}\right)$ for $\in\left[t_{k}^{x_{0}}, t_{k+1}^{x_{0}}\right)$.

This completes the proof. That is the $x$ remains in the open set $\mathbb{D}$. 


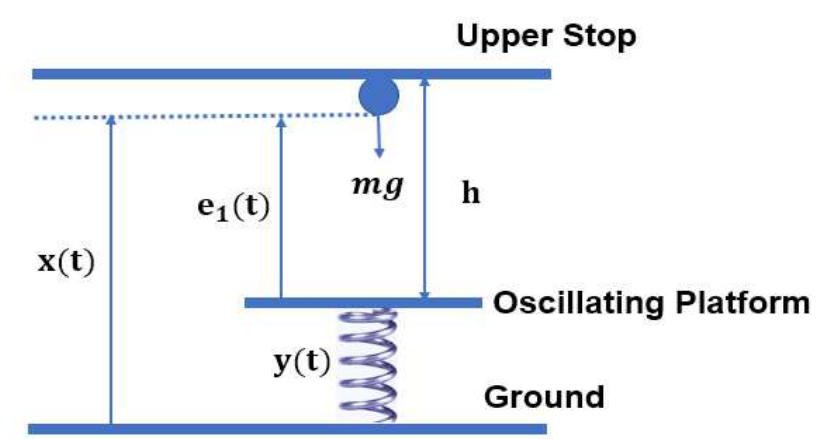

Fig. 1. Bouncing ball dynamics(model).

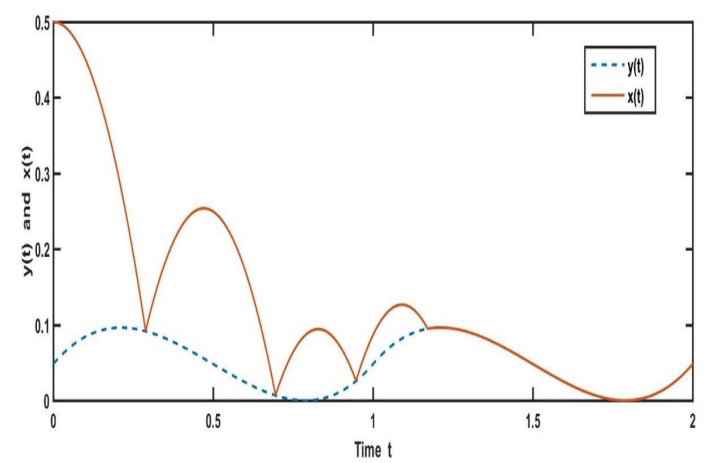

Fig. 2. Bouncing ball dynamics(trajectory).

Theorem 2. Let $\mathbb{D}$ is a bounded domain in $\mathbb{R}^{n}$, there exists a positive function $V(x)$ satisfy the following conditions:

$$
\left\{\begin{array}{l}
\left.D^{+} V(x)\right|_{(1)} \leq 0, x \in \mathbb{D} \\
V(x+S(x)) \leq V(x), x \in M
\end{array}\right.
$$

Then $x$ of system (1) remains in the open set $\mathbb{D}$.

Remark 1. In Theorem 1 and 2, for a given initial value $x_{0} \in \mathbb{D}$, the state of system (1) will remain in the set $\mathbb{D}$. The theory can be used to solve the bouncing ball system and the car collision problem $[32,33]$. These are typical state constrained hybrid systems. Such as the bouncing ball system:

Example 1. Let $x(t)$ be the displacement of the bal1, $y(t)$ be the displacement of the oscillating platform, $e_{1}(t)$ is relative displacement, $e_{2}(t)$ is relative velocity, $g$ is gravitational acceleration, $m$ be the mass of the ball and much less than that of the platform. When no collision occurs, the ball is only affected by gravity or moves with the platform. Assume that all collisions are instantaneous.

The bouncing ball dynamics can be modeled by

$$
\left\{\begin{array}{l}
\dot{e}_{1}(t)=e_{2}(t) \\
\dot{e}_{2}(t)=-g-\ddot{y}(t),\left(e_{1}(t), e_{2}(t)\right) \in \mathbb{D} \\
e_{2}\left(t^{+}\right)=-\mu e_{2}\left(t^{-}\right),\left(e_{1}(t), e_{2}(t)\right) \in M=\partial \mathbb{D}
\end{array}\right.
$$

Where $\mathbb{D}=\left\{\left(e_{1}(t), e_{2}(t)\right) \in \mathbb{R}^{2} \mid 0 \leq e_{1}(t) \leq h\right\}, M=$ $\left\{\left(e_{1}(t), e_{2}(t)\right) \in \mathbb{R}^{2} \mid e_{1}(t)=0\right.$ or $\left.h\right\}$, Consider a positive function $V(e(t))=\frac{1}{2} e_{2}^{2}(t)+g e_{1}(t)$, when $\left(e_{1}(t), e_{2}(t)\right) \notin$ $M$, the energy is decaying, then $\left.\dot{V}(e(t))\right|_{(4)} \leq 0$. When $\left(e_{1}(t), e_{2}(t)\right) \in M$, there has a collision and energy loss, that is $\mu \leq 1$, then $V\left(e\left(t^{+}\right)\right)=\frac{1}{2} e_{2}^{2}\left(t^{+}\right)+g e_{1}\left(t^{+}\right)=$ $\frac{1}{2} \mu^{2} e_{2}^{2}\left(t^{-}\right)+g e_{1}\left(t^{-}\right) \leq \frac{1}{2} 2 e_{2}^{2}\left(t^{-}\right)+g e_{1}\left(t^{-}\right)=V\left(e\left(t^{-}\right)\right)$. From Theorem 2, we can get that the ball will moved steadily with the platform (see fig. 2).

Remark 2. The bounce ball system is simple and fundamental, naturally associated with physics and engineering problems. In fact, it has been used as a simplified model for vibrating granular materials [34], heartbeat model. Moreover, the bouncing ball model is relevant to periodic excitation, such as moored ships driven by steady ocean waves $[32,33]$. The bounce ball system belongs to a typical class state constrained impulsive nonlinear systems.

\section{Control design for state constrained impulsive nonlinear dynamical networks}

In this paper, we consider the following nonlinear dynamical networks with linearly couplings:

$$
\dot{x}_{i}(t)=E x_{i}(t)+A g\left(x_{i}(t)\right)+c \sum_{j=1, j \neq i}^{N} b_{i j} \Gamma\left(x_{j}(t)-x_{i}(t)\right),
$$

where $i=1,2, \ldots, N . x_{i}(t)=\left(x_{i 1}(t), x_{i 2}(t), \ldots, x_{i n}(t)\right)^{T} \subset$ $\mathbb{R}^{n}$ is the state vector of the $i$-th node. And the state is constrained in $\mathbb{R}^{n}$ inside a ellipsoid $\Theta . g\left(x_{i}(t)\right)=$ $\left(g_{1}\left(x_{i 1}(t)\right), \ldots, g_{n}\left(x_{i n}(t)\right)\right)^{T}$ and $g_{j}: \mathbb{R}^{n} \rightarrow \mathbb{R}^{n}$ is a smooth nonlinear continuous functions. It is defined by $\mathcal{L}=\left\{g_{i}(\cdot) \mid g_{i} \in C\left(\mathbb{R}^{n}, \mathbb{R}^{n}\right), \exists L_{i}>0,0<s g_{i}(s) \leq\right.$ $\left.L_{i} s^{2}, \forall s \in \mathbb{R}, i=1,2, \ldots, n\right\}$. Let $L=\operatorname{diag}\left\{L_{1}, L_{2}, \ldots, L_{n}\right\}$. $c$ is alterable coupling strength. $E, A \in \mathbb{R}^{n \times n}$. $\Gamma$ is a positive definite diagonal matrix which describes the individual couplings between node $i$ and $j . B=\left(b_{i j}\right)_{n \times n}$ and $b_{i j}$ is defined as follows: if there is a connection from node $j$ to node $i(i \neq j)$ then $b_{i j}>0$; otherwise, $b_{i j}=0$, and $b_{i i}=-\sum_{j=1, j \neq i}^{N} b_{i j}$. For dynamical systems (5), its initial conditions are given by $x_{i}\left(t_{0}\right)=\phi_{i}\left(t_{0}\right) \in \Theta$. In addition, too much cohesion and the system suffers all the ills of complexity, such as the impulsive influence. In this paper, we assume that the impulses are produced under certain environment and condition. 
Then the system can be re-described by

$$
\left\{\begin{array}{l}
\dot{x}(t)=I_{N} \otimes E x(t)+I_{N} \otimes A g(x(t)) \\
+c(B \otimes \Gamma) x(t)+U(t), x(t) \in \Theta \\
\triangle x(t)=S(x(t)), x(t) \in M \subset \partial\left(\mathbb{R}^{n N} / \Theta\right) \\
x\left(t_{0}\right)=\phi\left(t_{0}\right)
\end{array}\right.
$$

Where $U(t)$ is the control input. $S(x(t))$ denotes the change at each impulsive instant.

It is not difficult to see that there exists a family of ellipsoids and set a positive definite matrix $R$ such that $\Theta:=\left\{x \mid x^{T} R x \leq 1\right\}$. In this paper, design the controller

$$
U(t)=\left\{\begin{array}{l}
-K x(t), x(t) \in \Theta, \\
0, x(t) \in M \subset \partial \Theta .
\end{array}\right.
$$

where $K$ is a gain matrix. Also, we need the following assumption and definition.

H1. Assume that there exists a constant $\mu \leq 1$ such that

$$
(x(t)+S(x(t)))^{T}(x(t)+S(x(t))) \leq \mu x^{T}(t) x(t) .
$$

In order to better study its properties, we introduce the definition of barrier Lyapunov functions in the following.

Definition 1. [25] A BLF is a scalar function $V(x)$, defined with respect to the system $\dot{x}=f(x)$ on an open region $\mathbb{D}$ containing the origin, that is continuous, positive definite, has continuous first-order partial derivatives at every point of $\mathbb{D}$, has the property $V(x) \rightarrow \infty$ as $x$ approaches the boundary of $\mathbb{D}$, and satisfies $V(x) \leq b$, $\forall t \geq 0$ along the solution $\dot{x}=f(x)$ for $x(0) \in \mathbb{D}$ and some positive constant $b$.

Remark 3. In fact, by definition, it is difficult to construct the appropriate barrier Lyapunov functions for the explicit model, especially for the impulsive model. In the following, the further analysis will be done for the state constrained impulsive systems.

Lemma 1. For any $\varepsilon>0, a \in \mathbb{R}^{n}, b \in \mathbb{R}^{n}$, the inequality $2 a^{T} b \leq \varepsilon a^{T} a+\varepsilon^{-1} b^{T} b$ holds.

In the following, we will present some stable criteria for systems (6) by different barrier Lyapunov functions.

Theorem 3. Under (H1), if there exists a matrix $0<P \in \mathbb{R}^{n \times n}$ and some constants $\gamma, \varepsilon_{1}, \varepsilon_{2} \in \mathbb{R}^{+}$such the following inequation holds:

$\Omega=\left(\begin{array}{cc}\Pi_{11}+\gamma I_{N} \otimes P & \Pi_{12} \\ \Pi_{12}^{T} & \Pi_{22}\end{array}\right) \leq 0$

Then, any state of the systems (6) start in the ellipsoidal set and remain in the set $\Theta$, and converge to zero. Where $\Pi_{11}=2 I_{N} \otimes P E+\varepsilon_{1} I_{N} \otimes P A A^{T} P-2 I_{N} \otimes$ $P K+\varepsilon_{1}^{-1} L^{2} I_{N \times n}+2 c(B \otimes P \Gamma)+\varepsilon_{2}^{-1} L^{2} I_{N \times n}-\gamma I_{N} \otimes R$. $\Pi_{12}=I_{N} \otimes R-I_{N} \otimes P+I_{N} \otimes E^{T} R^{T}+c\left(B^{T} \otimes \Gamma R\right)$. $\Pi_{22}=-2 I_{N} \otimes R K-2 I_{N} \otimes R+\varepsilon_{2} I_{N} \otimes R A A^{T} R$.
Proof. Consider the following BLF candidate

$$
V(x(t))=\log \left(\frac{1}{1-x^{T}(t) I_{N} \otimes R x(t)}\right) .
$$

In order to introduce the matrix $P$, which characterize the attractive ellipsoid, instead of substituting $\dot{x}$ as usual we use the descriptor method. Add the term

$$
\begin{gathered}
\Phi(x(t), \dot{x}(t))=\frac{1}{1-x^{T}(t) I_{N} \otimes R x(t)}\left(2 x^{T}(t) I_{N} \otimes P\right. \\
\left.+2 \dot{x}^{T}(t) I_{N} \otimes R\right)\left(I_{N} \otimes E x(t)+I_{N} \otimes A g(x(t))\right. \\
+c(B \otimes \Gamma) x(t)+U(t)-\dot{x}(t)) .
\end{gathered}
$$

We select the control input as linear input feedback $U(t)=-K x(t)$. When $x(t) \in \Theta$. The time derivative of $V(x)$ with respect to $t$ using (6) is given by $\left.\dot{V}(x(t))\right|_{(6)}$

$$
\begin{aligned}
= & \frac{2 x^{T}(t) I_{N} \otimes R \dot{x}(t)}{1-x^{T}(t) I_{N} \otimes R x(t)} \\
= & \frac{1}{1-x^{T}(t) I_{N} \otimes R x(t)}\left[2 x^{T}(t) I_{N} \otimes R \dot{x}(t)\right. \\
& +\left(2 x^{T}(t) I_{N} \otimes P+2 \dot{x}^{T}(t) I_{N} \otimes R\right)\left(I_{N} \otimes E x(t)\right. \\
& \left.+I_{N} \otimes A g(x(t))+c(B \otimes \Gamma) x(t)+U(t)-\dot{x}(t)\right] \\
= & \frac{1}{1-x^{T}(t) I_{N} \otimes R x(t)}\left[2 x^{T}(t) I_{N} \otimes R \dot{x}(t)\right. \\
& +2 x^{T}(t) I_{N} \otimes P E x(t)+2 x^{T}(t) I_{N} \otimes P A g(x(t)) \\
& +2 c x^{T}(t)(B \otimes P \Gamma) x(t)-2 x^{T}(t) I_{N} \otimes P K x(t) \\
& -2 x^{T}(t) I_{N} \otimes P \dot{x}(t)+2 \dot{x}^{T}(t) I_{N} \otimes R E x(t) \\
& +2 \dot{x}^{T}(t) I_{N} \otimes R A g(x(t))+2 c \dot{x}^{T}(t)(B \otimes R \Gamma) x(t) \\
& -2 \dot{x}^{T}(t) I_{N} \otimes R K x(t)-2 \dot{x}^{T}(t) I_{N} \otimes R \dot{x}(t) \\
\leq & \frac{1}{1-x^{T}(t) I_{N} \otimes R x(t)}\left[2 x^{T}(t) I_{N} \otimes R \dot{x}(t)\right. \\
& +2 x^{T}(t) I_{N} \otimes P E x(t)+\varepsilon_{1} x^{T}(t) I_{N} \otimes P A A^{T} P x(t) \\
& +\varepsilon_{1}^{-1} g^{T}(x(t)) g(x(t))+2 c x^{T}(t)(B \otimes P \Gamma) x(t) \\
& -2 x^{T}(t) I_{N} \otimes P K x(t)-2 x^{T}(t) I_{N} \otimes P \dot{x}(t) \\
& +2 \dot{x}^{T}(t) I_{N} \otimes R E x(t)+\varepsilon_{2} \dot{x}^{T}(t) I_{N} \otimes R A A^{T} R \dot{x}(t) \\
& +\varepsilon_{2}^{-1} g^{T}(x(t)) g(x(t))+2 c \dot{x}^{T}(t)(B \otimes R \Gamma) x(t) \\
& \left.-2 \dot{x}^{T}(t) I_{N} \otimes R K x(t)-2 \dot{x}^{T}(t) I_{N} \otimes R \dot{x}(t)\right] \\
&
\end{aligned}
$$

Let

$2 x^{T}(t) I_{N} \otimes R \dot{x}(t)+2 x^{T}(t) I_{N} \otimes P E x(t)+\varepsilon_{1} x^{T}(t) I_{N} \otimes$ $P A A^{T} P x(t)+\varepsilon_{1}^{-1} L^{2} x^{T}(t) x(t)+2 c x^{T}(t)(B \otimes P \Gamma) x(t)-$ $2 x^{T}(t) I_{N} \otimes P K x(t)-2 x^{T}(t) I_{N} \otimes P \dot{x}(t)+2 \dot{x}^{T}(t) I_{N} \otimes$ $R E x(t)+\varepsilon_{2} \dot{x}^{T}(t) I_{N} \otimes R A A^{T} R \dot{x}(t)+\varepsilon_{2}^{-1} L^{2} x^{T}(t) x(t)+$ $2 c \dot{x}^{T}(t)(B \otimes R \Gamma) x(t)-2 \dot{x}^{T}(t) I_{N} \otimes R K x(t)-2 \dot{x}^{T}(t) I_{N} \otimes$ $R \dot{x}(t) \leq-\gamma\left(1-x^{T}(t) I_{N} \otimes R x(t)\right)$

holds. That is, we can obtain that $\dot{V}(x) \leq-\gamma$, if $\delta^{T} \Omega_{0} \delta+$ $\gamma \leq 0$, where

$\Omega_{0}=\left(\begin{array}{ll}\Pi_{11} & \Pi_{12} \\ \Pi_{12}^{T} & \Pi_{22}\end{array}\right)$. 
Note $\delta=\left(x^{T}(t), \dot{x}^{T}(t)\right)^{T}$. We have

$\delta^{T} \Omega \delta+\gamma\left(1-x^{T}(t) I_{N} \otimes P x(t)\right) \leq 0$.

That is, if (7) holds, then the inequality (9) is implicit in the subset of $\mathbb{R}^{n}$ described by $\Theta$, then $\dot{V}(x) \leq$ $-\gamma<0$ for $x(t) \notin M$.

When $x(t) \in M$, under (H1), we have $V\left(x\left(t_{k}^{+}\right)\right)$

$$
\begin{aligned}
& =\log \left(\frac{1}{1-x^{T}\left(t_{k}^{+}\right) I_{N} \otimes R x\left(t_{k}^{+}\right)}\right) \\
& =\log \left(\frac{1}{1-\left[x\left(t_{k}^{-}\right)+S\left(x\left(t_{k}^{-}\right)\right)\right]^{T} I_{N} \otimes R\left[x\left(t_{k}^{-}\right)+S\left(x\left(t_{k}^{-}\right)\right)\right]}\right) \\
& \leq \log \left(\frac{1}{1-\mu x^{T}\left(t_{k}^{-}\right) I_{N} \otimes R x\left(t_{k}^{-}\right)}\right)
\end{aligned}
$$$$
\leq \log \left(\frac{1}{1-x^{T}\left(t_{k}^{-}\right) I_{N} \otimes R x\left(t_{k}^{-}\right)}\right)
$$$$
=V\left(t_{k}^{-}\right) \text {. }
$$

That is

$$
\left\{\begin{array}{l}
\left.D^{+} V(x)\right|_{(6)}<0, x \in \Theta \\
V\left(x\left(t_{k}^{+}\right)\right) \leq V\left(t_{k}^{-}\right), x \in M \subset \partial \Theta .
\end{array}\right.
$$

Combining with Theorem 2, the solution of the system (4) will attracted to zero. We get the conclusions. This completes the proof.

Remark 4. In the existing literature [35-40], there were few researches on the state constrained impulsive systems by barrier Lyapunov functions. In Theorem 3, symmetric bounded region are considered by appropriate symmetric barrier Lyapunov functions. However, for the asymmetric bounded region, we need consider the other different scaling functions. In the following, we assume that $x_{11}(t) \in\left(-d_{2}, d_{1}\right)$, in fact, in Theorem 2, $d_{1}=d_{2}$, the bound of state can be as a special suprasphere $\|x(t)\|<\alpha$. For the corresponding, there exist some positive constants $\eta_{1}, \eta_{2}$ such that

$$
\begin{gathered}
x^{T}(t) M x(t) \leq \eta_{1}, \forall x_{11}(t) \in\left[0, d_{1}\right) . \\
x^{T}(t) M x(t) \leq \eta_{2}, \forall x_{11}(t) \in\left[-d_{2}, 0\right) .
\end{gathered}
$$

\section{Lemma 2. Let}

$$
V_{2}(z)=q(z) \log \left(\frac{a}{a-\|z\|}\right)+(1-q(z)) \log \left(\frac{b}{b-\|z\|}\right),
$$

then the Lyapunov function candidate $V_{2}$ is positive definite and $z \in(-b, a)$. Where $a, b$ are some positive constants, and $q(z)=\left\{\begin{array}{l}1, \text { if } z>0, \\ 0, \text { if } z \leq 0 .\end{array}\right.$

Proof. For the sake of analysis, we can rewrite $V_{2}(x)$ as

$V_{2}(z)=\left\{\begin{array}{l}\log \left(\frac{a}{a-\|z\|}\right), \text { if } 0<z<a, \\ \log \left(\frac{b}{b-\|z\|}\right), \text { if }-b<z \leq 0 .\end{array}\right.$
For $-b<z<a$, we have that $V_{2}(z) \geq 0$ and that $V_{2}(z)=0$ if and only if $z=0$, that is $V_{2}(z)$ is positive definite and is a BLF.

Additionally, $V_{2}(z)$ is piecewise smooth within each of the two intervals $z \in(-b, 0)$ and $z \in[0, a)$. Together with the fact that $\lim _{z \rightarrow 0^{+}} \frac{d V_{2}(z)}{d z}=\lim _{z \rightarrow 0^{-}} \frac{d V_{2}(z)}{d z}=0$, we conclude that $z \in(-b, a)$.

Theorem 4. Under (H1), if there exists a matrix $0<P \in \mathbb{R}^{n \times n}$ and some constants $\gamma, \varepsilon_{1}, \varepsilon_{2}, \varepsilon_{3}, \varepsilon_{4} \in \mathbb{R}^{+}$ such the following inequations hold:

$\Omega_{1}=\left(\begin{array}{cc}\tilde{\Pi}_{11}+\gamma_{1} I_{N} \otimes P & \tilde{\Pi}_{12} \\ \tilde{\Pi}_{12}^{T} & \tilde{\Pi}_{22}\end{array}\right) \leq 0$

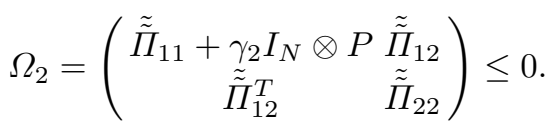

Then, any state of the systems (6) start in the ellipsoidal set and remain in the set, and converge to zero. Where $\tilde{\Pi}_{11}=2 I_{N} \otimes P E+\varepsilon_{1} I_{N} \otimes P A A^{T} P-2 I_{N} \otimes P K+$ $\varepsilon_{1}^{-1} L^{2} I_{N \times n}+2 c(B \otimes P \Gamma)+\varepsilon_{2}^{-1} L^{2} I_{N \times n}-\gamma_{1} I_{N} \otimes R$. $\tilde{\Pi}_{12}=q\left(x_{1}\right) I_{N} \otimes R-I_{N} \otimes P+I_{N} \otimes E^{T} R^{T}+c\left(B^{T} \otimes \Gamma R\right)$. $\tilde{\Pi}_{22}=-2 I_{N} \otimes R K-2 I_{N} \otimes R+\varepsilon_{2} I_{N} \otimes R A A^{T} R$. $\tilde{\tilde{I}}_{11}=2 I_{N} \otimes P E+\varepsilon_{3} I_{N} \otimes P A A^{T} P-2 I_{N} \otimes P K+$ $\varepsilon_{3}^{-1} L^{2} I_{N \times n}+2 c(B \otimes P \Gamma)+\varepsilon_{4}^{-1} L^{2} I_{N \times n}-\gamma_{2} I_{N} \otimes R$. $\tilde{\tilde{\Pi}}_{12}=\left(1-q\left(x_{1}\right)\right) I_{N} \otimes R-I_{N} \otimes P+I_{N} \otimes E^{T} R^{T}+c\left(B^{T} \otimes\right.$ $\Gamma R) . \tilde{\tilde{I}}_{22}=-2 I_{N} \otimes R K-2 I_{N} \otimes R+\varepsilon_{4} I_{N} \otimes R A A^{T} R$.

Proof. Consider the following BLF candidate

$$
\begin{aligned}
& V(x)=q(x) \log \left(\frac{\eta_{1}}{\eta_{1}-x^{T} I_{N} \otimes R x}\right) \\
& +(1-q(x)) \log \left(\frac{\eta_{2}}{\eta_{2}-x^{T} I_{N} \otimes R x}\right),
\end{aligned}
$$

where

$q(x)= \begin{cases}1, & \text { if } x_{11}>0 \\ 0, & \text { if } x_{11} \leq 0\end{cases}$

For easy of analysis, we can rewrite $V(x)$ as

$V(x)=\left\{\begin{array}{l}\log \left(\frac{\eta_{1}}{\eta_{1}-x^{T} I_{N} \otimes R x}\right), \text { if } 0<x_{11}<d_{1}, \\ \log \left(\frac{\eta_{2}}{\eta_{2}-x^{T} I_{N} \otimes R x}\right), \text { if }-d_{2}<x_{11} \leq 0 .\end{array}\right.$

In order to introduce the matrix $P$, which characterize the attractive ellipsoid, instead of substituting $\dot{x}$ as usual we use the descriptor method. Add the term

$$
\left\{\begin{array}{l}
\frac{1}{\eta_{1}-x^{T} I_{N} \otimes R x}\left(2 x^{T}(t) I_{N} \otimes P+2 \dot{x}^{T}(t) I_{N} \otimes R\right)\left(I_{N} \otimes E x(t)\right. \\
\left.+I_{N} \otimes A g(x(t))+c(B \otimes \Gamma) x(t)+U(t)-\dot{x}(t)\right), \\
\frac{1}{\eta_{2}-x^{T} I_{N} \otimes R x}\left(2 x^{T}(t) I_{N} \otimes P+2 \dot{x}^{T}(t) I_{N} \otimes R\right)\left(I_{N} \otimes E x(t)\right. \\
\left.+I_{N} \otimes A g(x(t))+c(B \otimes \Gamma) x(t)+U(t)-\dot{x}(t)\right) .
\end{array}\right.
$$


When $x(t) \in \Theta$, the time derivative of $V(x)$ is given by $\left.\dot{V}(x)\right|_{(6)}$

$$
\begin{aligned}
& =q(x) \frac{2 x^{T} I_{N} \otimes R \dot{x}}{\eta_{1}-x^{T} I_{N} \otimes R x}+(1-q(x)) \frac{2 x^{T} I_{N} \otimes R \dot{x}}{\eta_{2}-x^{T} I_{N} \otimes R x} \\
& =\frac{1}{\eta_{1}-x^{T} I_{N} \otimes R x}\left[2 q(x) x^{T}(t) I_{N} \otimes R \dot{x}(t)\right. \\
& +\left(2 x^{T}(t) I_{N} \otimes P+2 \dot{x}^{T}(t) I_{N} \otimes R\right)\left(I_{N} \otimes E x(t)\right. \\
& \left.+I_{N} \otimes A g(x(t))+c(B \otimes \Gamma) x(t)+U(t)-\dot{x}(t)\right] \\
& +\frac{1}{\eta_{2}-x^{T} I_{N} \otimes R x}\left[2(1-q(x)) x^{T}(t) I_{N} \otimes R \dot{x}(t)\right. \\
& +\left(2 x^{T}(t) I_{N} \otimes P+2 \dot{x}^{T}(t) I_{N} \otimes R\right)\left(I_{N} \otimes E x(t)\right. \\
& \left.+I_{N} \otimes A g(x(t))+c(B \otimes \Gamma) x(t)+U(t)-\dot{x}(t)\right] \\
& \leq \frac{1}{\eta_{1}-x^{T} I_{N} \otimes R x}\left[2 q(x) x^{T}(t) I_{N} \otimes R \dot{x}(t)\right. \\
& +2 x^{T}(t) I_{N} \otimes P E x(t)+\varepsilon_{1} x^{T}(t) I_{N} \otimes P A A^{T} P x(t) \\
& +\varepsilon_{1}^{-1} g^{T}(x(t)) g(x(t))+2 c x^{T}(t)(B \otimes P \Gamma) x(t) \\
& -2 x^{T}(t) I_{N} \otimes P K x(t)-2 x^{T}(t) I_{N} \otimes P \dot{x}(t) \\
& +2 \dot{x}^{T}(t) I_{N} \otimes R E x(t)+\varepsilon_{2} \dot{x}^{T}(t) I_{N} \otimes R A A^{T} R x(t) \\
& +\varepsilon_{2}^{-1} g^{T}(x(t)) g(x(t))+2 c \dot{x}^{T}(t)(B \otimes R \Gamma) x(t) \\
& \left.-2 \dot{x}^{T}(t) I_{N} \otimes R K x(t)-2 \dot{x}^{T}(t) I_{N} \otimes R \dot{x}(t)\right] \\
& +\frac{1}{\eta_{2}-x^{T} I_{N} \otimes R x}\left[2(1-q(x)) x^{T}(t) I_{N} \otimes R \dot{x}(t)\right. \\
& +2 x^{T}(t) I_{N} \otimes P E x(t)+\varepsilon_{3} x^{T}(t) I_{N} \otimes P A A^{T} P x(t) \\
& +\varepsilon_{3}^{-1} g^{T}(x(t)) g(x(t))+2 c x^{T}(t)(B \otimes P \Gamma) x(t) \\
& -2 x^{T}(t) I_{N} \otimes P K x(t)-2 x^{T}(t) I_{N} \otimes P \dot{x}(t) \\
& +2 \dot{x}^{T}(t) I_{N} \otimes R E x(t)+\varepsilon_{4} \dot{x}^{T}(t) I_{N} \otimes \\
& R A A^{T} R x(t)+\varepsilon_{4}^{-1} g^{T}(x(t)) g(x(t)) \\
& +2 c \dot{x}^{T}(t)(B \otimes R \Gamma) x(t)-2 \dot{x}^{T}(t) I_{N} \otimes R K x(t) \\
& \left.-2 \dot{x}^{T}(t) I_{N} \otimes R \dot{x}(t)\right]
\end{aligned}
$$

Let

$2 q(x) x^{T}(t) I_{N} \otimes R \dot{x}(t)+2 x^{T}(t) I_{N} \otimes P E x(t)+\varepsilon_{1} x^{T}(t) I_{N} \otimes$ $P A A^{T} P x(t)+\varepsilon_{1}^{-1} g^{T}(x(t)) g(x(t))+2 c x^{T}(t)(B \otimes P \Gamma) x(t)-$ $2 x^{T}(t) I_{N} \otimes P K x(t)-2 x^{T}(t) I_{N} \otimes P \dot{x}(t)+2 \dot{x}^{T}(t) I_{N} \otimes$ $R E x(t)+\varepsilon_{2} \dot{x}^{T}(t) I_{N} \otimes R A A^{T} R x(t)+\varepsilon_{2}^{-1} g^{T}(x(t)) g(x(t))+$ $2 c \dot{x}^{T}(t)(B \otimes R \Gamma) x(t)-2 \dot{x}^{T}(t) I_{N} \otimes R K x(t)-2 \dot{x}^{T}(t) I_{N} \otimes$ $R \dot{x}(t) \leq-\gamma_{1}\left(\eta_{1}-x^{T}(t) I_{N} \otimes R x(t)\right)$ and $2(1-q(x)) x^{T}(t) I_{N}$ $R \dot{x}(t)+2 x^{T}(t) I_{N} \otimes P E x(t)+\varepsilon_{3} x^{T}(t) I_{N} \otimes P A A^{T} P x(t)+$ $\varepsilon_{3}^{-1} g^{T}(x(t)) g(x(t))+2 c x^{T}(t)(B \otimes P \Gamma) x(t)-2 x^{T}(t) I_{N} \otimes$ $P K x(t)-2 x^{T}(t) I_{N} \otimes P \dot{x}(t)+2 \dot{x}^{T}(t) I_{N} \otimes R E x(t)+$ $\varepsilon_{4} \dot{x}^{T}(t) I_{N} \otimes R A A^{T} R x(t)+\varepsilon_{4}^{-1} g^{T}(x(t)) g(x(t))+2 c \dot{x}^{T}(t)(B$ $R \Gamma) x(t)-2 \dot{x}^{T}(t) I_{N} \otimes R K x(t)-2 \dot{x}^{T}(t) I_{N} \otimes R \dot{x}(t) \leq$ $-\gamma_{2}\left(\eta_{2}-x^{T}(t) I_{N} \otimes R x(t)\right)$ hold. That is, we can obtain that $\dot{V}(x) \leq-\gamma_{1} \eta_{1}-\gamma_{2} \eta_{2}$, if $\delta^{T} \tilde{\Omega}_{1} \delta+\delta^{T} \tilde{\Omega}_{2} \delta+\gamma_{1} \eta_{1}+$ $\gamma_{2} \eta_{2} \leq 0$, where

$\tilde{\Omega}_{1}=\left(\begin{array}{ll}\tilde{\Pi}_{11} & \tilde{\Pi}_{12} \\ \tilde{\Pi}_{12}^{T} & \tilde{\Pi}_{22}\end{array}\right), \quad \tilde{\Omega}_{2}=\left(\begin{array}{ll}\tilde{\tilde{\Pi}}_{11} & \tilde{\tilde{\Pi}}_{12} \\ \tilde{\tilde{\Pi}}_{12}^{T} & \tilde{\Pi}_{22}\end{array}\right)$.
Note $\delta=\left(x^{T}(t), \dot{x}^{T}(t)\right)^{T}$, we have

$$
\begin{gathered}
\delta^{T} \Omega_{1} \delta+\delta^{T} \Omega_{2} \delta+\gamma_{1}\left(\eta_{1}-x^{T}(t) I_{N} \otimes P x(t)\right) \\
+\gamma_{2}\left(\eta_{2}-x^{T}(t) I_{N} \otimes P x(t)\right) \leq 0 .
\end{gathered}
$$

That is, if (12) and (13) hold, then the above inequality is implicit in the subset of $\mathbb{R}^{n}$ described by $\Theta$, then $\dot{V}(x) \leq-\gamma<0$ for $x(t) \notin M$.

When $x(t) \in M$, under (H1), we have $V\left(x\left(t_{k}^{+}\right)\right)$

$$
\begin{aligned}
= & q\left(x\left(t_{k}^{+}\right)\right) \log \left(\frac{\eta_{1}}{\eta_{1}-x^{T}\left(t_{k}^{+}\right) I_{N} \otimes R x\left(t_{k}^{+}\right)}\right) \\
& +\left(1-q\left(x\left(t_{k}^{+}\right)\right)\right) \log \left(\frac{\eta_{2}}{\eta_{2}-x^{T}\left(t_{k}^{+}\right) I_{N} \otimes R x\left(t_{k}^{+}\right)}\right) \\
= & q\left(x\left(t_{k}^{+}\right)\right) \times \\
& \log \left(\frac{\eta_{1}}{\eta_{1}-\left[x\left(t_{k}^{-}\right)+S\left(x\left(t_{k}^{-}\right)\right)\right]^{T} I_{N} \otimes R\left[x\left(t_{k}^{-}\right)+S\left(x\left(t_{k}^{-}\right)\right)\right]}\right) \\
& +\left(1-q\left(x\left(t_{k}^{+}\right)\right)\right) \times \\
& \log \left(\frac{\eta_{2}}{\eta_{2}-\left[x\left(t_{k}^{-}\right)+S\left(x\left(t_{k}^{-}\right)\right)\right]^{T} I_{N} \otimes R\left[x\left(t_{k}^{-}\right)+S\left(x\left(t_{k}^{-}\right)\right)\right]}\right) \\
\leq & q\left(x\left(t_{k}^{+}\right)\right) \log \left(\frac{\eta_{1}}{\eta_{1}-\mu x^{T}\left(t_{k}^{-}\right) I_{N} \otimes R x\left(t_{k}^{-}\right)}\right) \\
& +\left(1-q\left(x\left(t_{k}^{+}\right)\right)\right) \log \left(\frac{\eta_{2}}{\eta_{2}-\mu x^{T}\left(t_{k}^{-}\right) I_{N} \otimes R x\left(t_{k}^{-}\right)}\right) \\
\leq & q\left(x\left(t_{k}^{+}\right)\right) \log \left(\frac{\eta_{1}}{\eta_{1}-x^{T}\left(t_{k}^{-}\right) I_{N} \otimes R x\left(t_{k}^{-}\right)}\right) \\
& +\left(1-q\left(x\left(t_{k}^{+}\right)\right)\right) \log \left(\frac{\eta_{2}}{\eta_{2}-x^{T}\left(t_{k}^{-}\right) I_{N} \otimes R x\left(t_{k}^{-}\right)}\right) \\
= & V\left(t_{k}^{-}\right) .
\end{aligned}
$$

This completes the proof.

Remark 5. Compared with traditional Lyapunov function [41-43], it is difficult to calculate theoretically for barrier Lyapunov function. For the asymmetric problems, this is a tough one to solve. Theorem 3 and 4 provide a reference in theoretical result and analyzed method for the constrained systems. In the future, we continue to find a better way to consider it.

Remark 6. In this paper, we focus on the state constraint systems with impulsive input effects. The general assumption for impulsive input is provided for a potentially large control effort is key to safeguarding against any constraint transgression. Nevertheless, the trajectory of system remains bounded for all time. By careful selection of control parameters, we can limit the control signal within a desirable operating range or stabilize the state of systems.

\section{Examples}

In this section, we will provide a numerical example to illustrate the effectiveness of the proposed criteria in this paper. 
Example 1. Consider the following nonlinear dynamic networks with 3 -nodes

$$
\left\{\begin{array}{l}
\dot{x}(t)=I_{N} \otimes E x(t)+I_{N} \otimes A g(x(t)) \\
+c(B \otimes \Gamma) x(t)+U(t), x(t) \in \mathbb{D} \\
\triangle x(t)=S(x(t)), x(t) \in M \subset \partial \mathbb{D} \\
x\left(t_{0}\right)=\phi\left(t_{0}\right)
\end{array}\right.
$$

Where

$$
\begin{aligned}
& x(t)=\left(x_{1}(t), x_{2}(t), x_{3}(t)\right)^{T} \in \mathbb{D}, \\
& \mathbb{D}=\left\{x_{i j}(t) \in \mathbb{R}|| x_{i j}(t) \mid<1.2, i=1,2,3 . j=1,2\right\}, \\
& x_{i}(t)=\left(x_{i 1}(t), x_{i 2}(t)\right)^{T}, \\
& E=\left(\begin{array}{ll}
1.1 & 0.2 \\
0.1 & 1.3
\end{array}\right), A=\left(\begin{array}{ll}
1.5 & 1.1 \\
0.9 & 1.4
\end{array}\right) \\
& B=\left(\begin{array}{cc}
1 & -1 \\
-1 & 1
\end{array}\right), \Gamma=\left(\begin{array}{lll}
2 & 0 & 0 \\
0 & 1 & 0 \\
0 & 0 & 3
\end{array}\right) \\
& M=\left\{x_{i j}(t) \in \mathbb{R}|| x_{i j}(t) \mid=1.2, i=1,2,3 . j=1,2\right\}, \\
& g(s)=\frac{|s+1|+|s-1|}{2}, \\
& \triangle x(t)=-0.65 x_{1}(t) .
\end{aligned}
$$

Here, the initial condition of systems (17) is chosen as: $x(0)=(1.05 ; 1.07 ; 1.15 ;-1.15 ;-0.9 ;-0.78)^{T}$. Fig. $3-5$ show the state trajectories for system (17) without control input. From Fig. 3, we can see that when $x_{11}(t)=1.2$, it will be triggered the impulsive mechanism. From Fig. 4, when $x_{21}(t)=-1.2$ 0r $x_{22}(t)=$ -1.2 , system (17) will be triggered the impulsive mechanism. The shape of Fig. 5 changed depending on Fig. 3 and 4 . In conclusion, the state trajectories for systems (17) never going out of a certain range.

Design $U(t)=K x(t)=-5.5 x(t)$. Choose $\varepsilon_{1}=0.95$, $\varepsilon_{2}=0.95, R=2 I$. By numerical count software MATLAB LMI Toolbox, we can get that

$$
P=\left(\begin{array}{ll}
1.9479 & 0.7683 \\
0.7683 & 1.8925
\end{array}\right)
$$

So the conditions of Theorem 3 are all satisfied and dynamic systems (17) is stable. Fig. 6 shows the time response of states of (17) with controller.

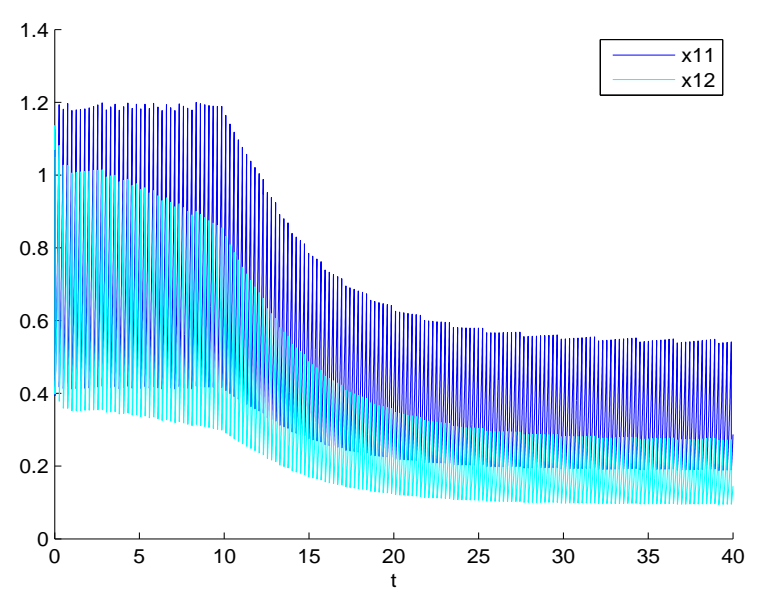

Fig. 3. Transient response of state variable $x(t)$ of system (17) without controller.

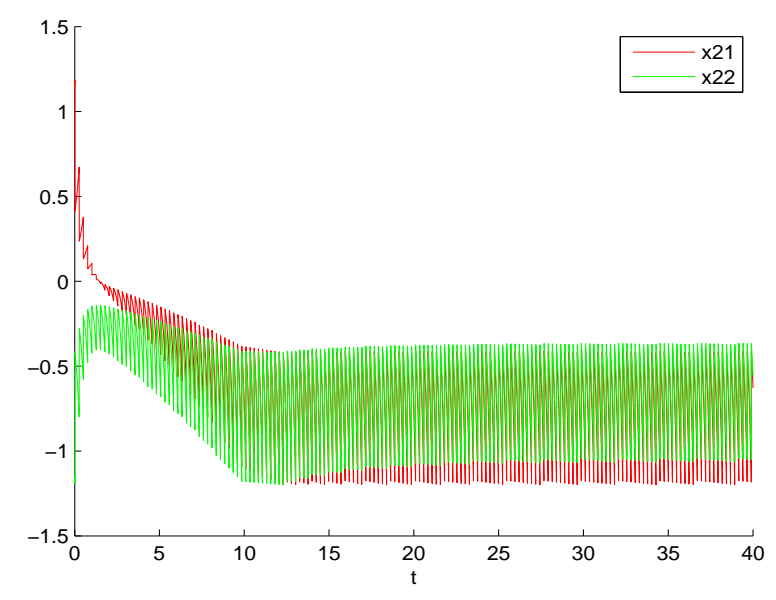

Fig. 4. Transient response of state variable $x(t)$ of system (17) without controller.

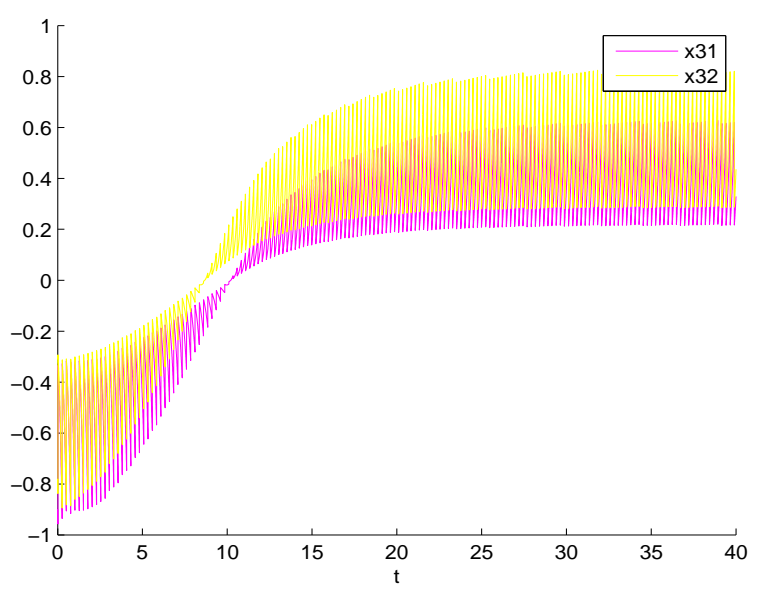

Fig. 5. Transient response of state variable $x(t)$ of system (17) without controller. 


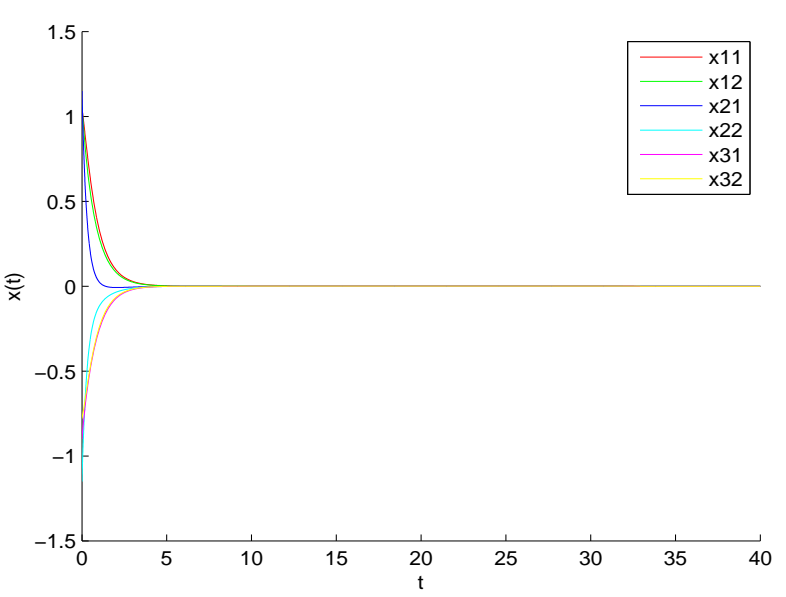

Fig. 6. Transient response of state variable $x(t)$ of system (17) with controller.

\section{Conclusions}

This paper consider the problems of state constrained nonlinear systems via attractive ellipsoid method, auxiliary matrix and barrier Lyapunov method, some stability criteria have been derived. In addition, the usefulness of the proposed results has been demonstrated by one numerical example. In our future work, designing a state constrained impulsive control law for a class of delayed dynamic systems by using the proposed methodology (or other barrier Lyapunov functions) will be considered. In conclusion, there is always something more challenging for the problem of state constrained system.

Acknowledgements This study was funded by Natural Science Foundation Project of Anhui, and in part by the University Natural Science Research Project of Anhui Province under Grant KJ2020ZD008, in part by the National Natural Science Foundation of China under Grant 61873213, and in part by the Natural Science Foundation Project of Chongqing under grants Nos. cstc2018jcyjAX0588.

\section{Data availability statement}

The datasets generated during and/or analyzed during the current study are available from the corresponding author on reasonable request.

\section{Declarations}

\section{Conflict of interest}

The authors declare that they have no conflict of interest.

\section{References}

1. Simon D. Kalman filtering with state constraints: a survey of linear and nonlinear algorithms. IET Control Theory \& Applications, 2010, 4(8): 1303-1318.

2. Du H, Zhang N. Fuzzy Control for Nonlinear Uncertain Electrohydraulic Active Suspensions With Input Constraint. IEEE Transactions on Fuzzy Systems, 2009, 17(2): 343356.

3. Zhou J, Wen C, Zhang Y, et al. Adaptive output control of nonlinear systems with uncertain dead-zone nonlinearity. IEEE Transactions on Automatic Control, 2006, 51(3): 504511.

4. He W, Dong Y, Sun C. Adaptive Neural Impedance Control of a Robotic Manipulator With Input Saturation. IEEE Transactions on systems man and cybernetics, 2016, 46(3):334-344.

5. Guo H, Chen L. Time-limited pest control of a LotkaCVolterra model with impulsive harvest. Nonlinear Analysis: Real World Applications, 2009, 10(2): 840-848.

6 . Korn R. Some applications of impulse control in mathematical finance. Mathematical Methods of Operations Research, 1999, 50(3): 493-518.

7. Button M, Gardiner J, Glover I. Measurement of the impulsive noise environment for satellite-mobile radio systems at $1.5 \mathrm{GHz}$. IEEE Transactions on Vehicular Technology, 2002, 51(3): 551-560.

8. Dashkovskiy S, Mironchenko A. Input-to-state stability of nonlinear impulsive systems. SIAM Journal on Control and Optimization, 2013, 51(3): 1962-1987.

9. Li X, Ho DWC, Cao J. Finite-time stability and settlingtime estimation of nonlinear impulsive systems. Automatica, 2019, 99: 361-368.

10. Li X, Song S. Stabilization of delay systems: delaydependent impulsive control. IEEE Transactions on Automatic Control, 2016, 62(1): 406-411.

11. Li X, Li P, Wang Q. Input/output-to-state stability of impulsive switched systems. Systems \& Control Letters, 2018, 116: $1-7$.

12. Wang J, Liang J, Qiu J. Asynchronous 11 control for $2 \mathrm{D}$ switched positive systems with parametric uncertainties and impulses. Nonlinear Analysis: Hybrid Systems, 2020, 37:100887.

13. Li, L, Wang, X, Li, C, Feng, Y. Exponential synchronizationlike criterion for state-dependent impulsive dynamical networks. IEEE Transactions on Neural Networks, 2019, 30(4): 1025-1033.

14. Zamani I, Shafiee M, Ibeas A. On singular hybrid switched and impulsive systems. International Journal of Robust and Nonlinear Control, 2018, 28(2): 437-465.

15. Guan Z, Hill D J, Shen X. On hybrid impulsive and switching systems and application to nonlinear control. IEEE Transactions on Automatic Control, 2005, 50(7): 1058-1062.

16. Rao C, Rawlings J, Mayne D. Constrained state estimation for nonlinear discrete-time systems: stability and moving horizon approximations. IEEE Transactions on Automatic Control, 2003, 48(2): 246-258. 
17. Harris M, Acikmese B. Lossless convexification of nonconvex optimal control problems for state constrained linear systems. Automatica, 2014 50(9):2304-2311.

18. Ngo K, Mahony R, Jiang Z. Integrator Backstepping using Barrier Functions for Systems with Multiple State Constraints. Conference on Decision and Control, 2005: 83068312.

19. Dehaan D, Guay M. Extremum-seeking control of stateconstrained nonlinear systems. Automatica, 2005, 41(9): 1567-1574.

20. Li L, Li C, Zhang W. Delayed-impulsive control for difference systems with actuator saturation and its synchronisation application. IET Control Theory \& Applications, 2019, 13(8): 1129-1136.

21. Lampart M, Zapoml J. Chaos identification of a colliding constrained body on a moving belt. Nonlinear Dynamics, 2021, 104: 2723-2732.

22. Wu Z, Albalawi F, Zhang Z, Zhang J, et al. Control Lyapunov-Barrier function-based model predictive control of nonlinear systems. Automatica, 2019, 109(109).

23. Li L, Li C, Li H. Fully state constraint impulsive control for non-autonomous delayed nonlinear dynamic systems. Nonlinear Analysis: Hybrid Systems, 2018, 29: 383-394.

24. Liu Y, Tong S. Barrier Lyapunov Functions-based adaptive control for a class of nonlinear pure-feedback systems with full state constraints. Automatica, 2016, 64(64): 70-75.

25. Tee K, Ren B, Ge B. Control of nonlinear systems with time-varying output constraints. Automatica, 2011, 47(11): 2511-2516.

26. Liu C, Dong C, Zhou Z, Wang Z. Barrier Lyapunov function based reinforcement learning control for air-breathing hypersonic vehicle with variable geometry inlet. Aerospace Science and Technology, 2020. 96:105537.

27. Liu X, Chen D, Liu Z, Wang Y. Distributed leaderless impulsive consensus of non-linear multi-agent systems with input saturation. Nonlinear Analysis: Hybrid Systems, 2020, 36:100855.

28. Ma L, Huo X, Zhao X, Zong G. Observer-based adaptive neural tracking control for output-constrained switched MIMO nonstrict-feedback nonlinear systems with unknown dead zone. Nonlinear Dynamics, 2020, 99: 1019-1036.

29. Yao Y, Tan J, Wu J, Zhang X. Event-triggered fixedtime adaptive fuzzy control for state-constrained stochastic nonlinear systems without feasibility conditions. Nonlinear Dynamics volume, 2021, 105: 403-416.

30. Rakovic S, Kerrigan E, Kouramas K, Mayne D. Invariant approximations of the minimal robust positively invariant set. IEEE Transactions on Automatic Control, 2005, 50(3): 406-410.

31. P. Trodden, A one-step approach to computing a polytopic robust positively invariant set. IEEE Transactions on Automatic Control, 2016, 61(12): 4100-4105.

32. Leine R, Heimsch T. Global uniform symptotic attractive stability of the non-autonomous bouncing ball system. Physica D: Nonlinear Phenomena, 2012;241(22):2029-2041.

33. Barroso J, Carneiro M, Macau E. Bouncing ball problem: stability of the periodic modes. Physical Review E, 2009, 79(2): 026206.

34. Pastor J M, Maza D, Zuriguel I, et al. Time resolved particle dynamics in granular convection. Physica D: Nonlinear Phenomena, 2007, 232(2): 128-135.

35. Bemporad A, Morari M, Dua V, et al. The explicit linear quadratic regulator for constrained systems. Automatica, 2002, 38(1): 3-20.

36. Li D, Liu Y, Tong S, Chen C, Li D. Neural networksbased adaptive control for nonlinear state constrained systems with input delay. IEEE Transactions on Systems, Man, and Cybernetics, 2018 49(4): 1249-1258.
37. Chen Y, Wang Z, Shen B, Dong H. Exponential synchronization for delayed dynamical networks via intermittent control: dealing with actuator saturations. IEEE Transactions on Neural Networks, 2019, 30(4): 1000-1012.

38. Yu J, Zhao L, Yu H, Lin C. Barrier Lyapunov functionsbased command filtered output feedback control for fullstate constrained nonlinear systems. Automatica, 2019, 105: 71-79.

39. Liu Y, Lu S, Tong S, Chen X, Chen C, Li, D. Adaptive control-based Barrier Lyapunov Functions for a class of stochastic nonlinear systems with full state constraints. Automatica, 2018, 87: 83-93.

40. Liu Y, Tong S. Barrier Lyapunov functions-based adaptive control for a class of nonlinear pure-feedback systems with full state constraints. Automatica, 2016, 64: 70-75.

41. Johnson E, Calise A. Neural network adaptive control of systems with input saturation. Proceedings of the American Control Conference, 2001, 5: 3527-3532.

42. Liu Y, Tong S. Barrier Lyapunov functions for Nussbaum gain adaptive control of full state constrained nonlinear systems. Automatica, 2017, 76: 143-152.

43. Tee K, Ge S. Control of nonlinear systems with partial state constraints using a barrier Lyapunov function. International Journal of Control, 2011, 84(12): 2008-2023. 